\title{
INVARIANT EIGENDISTRIBUTIONS ON SEMISIMPLE LIE GROUPS
}

\author{
BY HARISH-CHANDRA ${ }^{1}$
}

Communicated by Raoul Bott, September 13, 1962.

1. Let $M$ be an oriented separable differentiable manifold of dimension $n$. (We do not assume that $M$ is connected.) Let $C_{c}^{\infty}(M)$ denote the space of all complex-valued $C^{\infty}$ functions on $M$ with compact support. A distribution $T$ on $M$ is a linear mapping $T: C_{c}^{\infty}(M) \rightarrow C$ which is continuous in the topology of Schwartz. More explicitly, this means the following. Let $U$ be any open and relatively compact set in $M$. Then we can select differential operators ${ }^{2} D_{1}, \cdots, D_{r}$ on $M$ such that

$$
|T(f)| \leqq \sum_{i} \sup \left|D_{i} f\right| \quad\left(f \in C_{c}^{\infty}(U)\right) .
$$

Let $G$ be a group acting on $M$. We denote by $x^{g}$ the transform of $x \in M$ by $g \in G$. We assume that, for a fixed $g$, the mapping $x \rightarrow x^{g}$ of $M$ is of class $C^{\infty}$. Then for any $f \in C_{c}^{\infty}(M)$, the function $f^{a}: x \rightarrow f\left(x^{g^{-1}}\right)$ is again in $C_{c}^{\infty}(M)$ and if $T$ is a distribution, the mapping $T^{0}$ : $f \rightarrow T\left(f^{o^{-1}}\right)\left(f \in C_{c}^{\infty}(M)\right)$ is also a distribution. We say $T$ is invariant (under $G$ ) if $T^{o}=T$ for all $g \in G$.

Now $G$ operates in a natural way on the spaces ${ }^{2}$ of differential operators and differential forms on $M$. For example if $D$ is a differential operator, $D^{o} f=\left(D f^{\sigma^{-1}}\right)^{\circ}\left(f \in C_{c}^{\infty}(M), g \in G\right)$. Fix a (real) differential form $\omega$ on $M$ of degree $n$ which is invariant under $G$ and which is everywhere positive (with respect to the given orientation of $M$ ). Then for every differential operator $D$ on $M$, we define its adjoint $D^{*}$ to be the (unique) differential operator satisfying the relation

$$
\int_{M} D f \cdot \phi \omega=\int_{M} f D^{*} \phi \cdot \omega
$$

for all $f, \phi \in C_{c}^{\infty}(M)$. If $T$ is a distribution, the mapping $f \rightarrow T\left(D^{*} f\right)$ $\left(f \in C_{c}^{\infty}(M)\right)$ is also a distribution which we denote by $D T$. Now $\omega$ defines a positive Borel measure $\mu$ on $M$. For example if $U$ is an open set in $M$,

1 This work was supported by a grant from the Sloan foundation and a contract with the U. S. Army.

${ }^{2}$ All differential operators and differential forms are meant to be $C^{\infty}$ unless explicitly mentioned otherwise. 


$$
\mu(U)=\int_{U} \omega .
$$

Let $F$ be a function on $M$ which is locally summable (with respect to $\mu$ ). Then corresponding to $F$, we get a distribution

$$
T_{F}: f \rightarrow \int f F d \mu=\int_{M} f F \cdot \omega \quad\left(f \in C_{c}^{\infty}(M)\right) .
$$

If $T$ is a distribution, we say $T=F$ if $T=T_{F}$.

2. Let $G$ be a connected semisimple Lie group. Take $M=G$, $x^{g}=g x g^{-1}(x, g \in G)$ and $\omega$ the invariant differential form corresponding to the Haar measure $d x$ on $G$. Let 8 be the algebra of all differential operators on $G$ which are invariant under both left and right translations of $G$. Then 8 is abelian. Let $l=\operatorname{rank} G$. $t$ being an indeterminate, we denote by $D(x)$ the coefficient of $t^{l}$ in $\operatorname{det}(t+1-\operatorname{Ad}(x))(x \in G)$. Then $D$ is an analytic function on $G$ and an element $x \in G$ is called regular if $D(x) \neq 0$. Let $G^{\prime}$ be the set of all regular elements in $G$. Then $G^{\prime}$ is an open and dense subset of $G$ whose complement is of measure zero.

Let $\Theta$ be a distribution on $G$. We say that it is invariant if $\Theta^{x}$ $=\Theta(x \in G)$ and that it is an eigendistribution of 8 if $z \Theta=\chi(z) \Theta(z \in Z)$ for some homomorphism $\chi$ of $\mathbf{Z}$ into $\boldsymbol{C}$.

TheOREM 1. Let $\Theta$ be an invariant eigendistribution of 8 on $G$. Then $\Theta$ is a locally summable function which is analytic on $G^{\prime}$.

This answers, in particular, a question raised in [3, p. 396].

3. Now assume that the center of $G$ is finite. Fix a maximal compact subgroup $K$ of $G$ and let $\varepsilon_{K}$ denote the set of all equivalence classes of irreducible finite-dimensional representations of $K$. For any $\mathfrak{b} \in \varepsilon_{K}$, let $\xi_{\mathrm{b}}$ be the character of $\mathfrak{d}$ and $\delta^{*}$ the class contragradient to D so that ${ }^{3} \xi_{\phi^{*}}(k)=\operatorname{conj} \xi_{\triangleright}(k)(k \in K)$. For any $f \in C_{c}^{\infty}(G)$, define

$$
f_{\mathfrak{b}}(x)=d\left(\wp^{\prime}\right) \int_{K} \xi_{\mathfrak{b}}(k) f(k x) d k \quad(x \in G),
$$

where $d(\mathfrak{b})$ is the degree of any representation in the class $b$ and $d k$ is the normalized Haar measure of $K$. Then $f_{b} \in C_{c}^{\infty}(G)$ and the series $\sum_{b \in \mathcal{E}_{K}} f_{b}$ converges in $C_{c}^{\infty}(G)$ to $f$. If $T$ is any distribution on $G$, the mapping $f \rightarrow T\left(f_{b^{*}}\right)\left(f \in C_{c}^{\infty}(G)\right)$ is also a distribution, which we denote by $T_{\mathrm{b}}$. Since

\footnotetext{
${ }^{3}$ conj $c$ stands for the complex conjugate for $c \in C$.
} 


$$
T(f)=\sum_{\mathfrak{b} \in \mathcal{E}_{K}} T_{\mathfrak{b}}(f)
$$

it is clear that $T_{\triangleright} \neq 0$ for some $\delta \in \varepsilon_{K}$, if $T \neq 0$.

Now suppose $T$ is an eigendistribution of 3 on $G$. Then the same holds for $T_{\mathfrak{b}}\left(b \in \varepsilon_{K}\right)$. But since $T_{\mathfrak{b}}$ transforms, under left translations by elements of $K$, according to $\delta$, it follows easily that it satisfies an elliptic differential equation on $G$ with analytic coefficients. Therefore $T_{\mathrm{b}}$ is an analytic function.

4. Let $\mathrm{g}$ be the Lie algebra of $G$ and $\mathfrak{g}_{c}$ its complexification. Let $G_{c}$ be the simply connected complex-analytic group corresponding to $\mathfrak{g}_{c}$. Assume that $G$ is the real analytic subgroup of $G_{c}$ corresponding to $\mathrm{g}$ and $\operatorname{rank} G=\operatorname{rank} K$. Fix a maximal connected abelian subgroup $A$ of $K$ and let $a$ denote its Lie algebra. Then $A$ is a Cartan subgroup of $G$ and $A^{\prime}=A \cap G^{\prime}$ is open and dense in $A$. Let $\mathfrak{a}_{c}$ denote the complexification of $\mathfrak{a}, P$ the set of all positive roots (under some fixed order) and $W$ the Weyl group of $\left(\mathfrak{g}_{c}, \mathfrak{a}_{c}\right)$. Then there exists an analytic function $\Delta$ on $A$ such that

$$
\Delta(\exp H)=\prod_{\alpha \in P}\left(e^{\alpha(H) / 2}-e^{-\alpha(H) / 2}\right) \quad(H \in \mathfrak{a}) .
$$

Let $\hat{A}$ denote the character group of $A$. For any $\hat{a} \in \hat{A}$, define

$$
\sigma(\hat{a})=\prod_{\alpha \in P}\langle\alpha, \lambda\rangle
$$

where $\lambda$ is the linear function on $\mathfrak{a}_{c}$ such that $\hat{a}(\exp H)=e^{\lambda(H)}(H \in \mathfrak{a})$ and $\langle\alpha, \lambda\rangle$ denotes the usual scalar product defined under the Killing form of $g_{c}$. $W$ operates on $\hat{A}$ in a natural way by duality. An element $\hat{a} \in \hat{A}$ is called regular if its transforms $\hat{a}^{s}(s \in W)$ are all distinct. Then $\hat{a}$ is singular or regular according as $\sigma(\hat{a})=0$ or not. Moreover $\sigma\left(\hat{a}^{s}\right)$ $=\epsilon(s) \sigma(\hat{a})(s \in W, \hat{a} \in \hat{A})$, where $\epsilon(s)=1$ or -1 and is independent of $\hat{a}$.

If $\Theta$ is an invariant eigendistribution of 8 on $G$, one can, in view of Theorem 1, speak of the value $\Theta(x)$ of $\Theta$ at any point $x \in G^{\prime}$. Define the function $D$ as in $\$ 2$.

THEOREM 2. Fix a regular element $\hat{a} \in \hat{A}$. Then there exists exactly one invariant eigendistribution $\Theta_{a}$ of $B$ on $G$ such that:

(1) The function $|D|^{1 / 2} \Theta_{d}$ remains bounded on $G^{\prime}$;

(2) $\Theta_{d}=(-1)^{q} \sigma(\hat{a}) \Delta^{-1} \sum_{s \in W} \epsilon(s) \hat{a}^{s}$ pointwise on $A^{\prime}$. Here $q=\frac{1}{2}(\operatorname{dim} G-\operatorname{dim} K)$.

For $f, g \in C_{c}^{\infty}(G)$, let $f * g$ denote their convolution product so that 


$$
(f * g)(x)=\int_{G} f(y) g\left(y^{-1} x\right) d y \quad(x \in G) .
$$

Also let $\tilde{f}(x)=\operatorname{conj}\left(f\left(x^{-1}\right)\right)$.

Theorem 3. Put $\Theta=\Theta_{a}$ for a fixed regular element $\hat{a}$ in $\hat{A}$. Then $\Theta(\tilde{f} * f) \geqq 0$ for every $f \in C_{c}^{\infty}(G)$. Moreover the analytic functions $\Theta_{\mathrm{b}}\left(\boldsymbol{b} \in \mathcal{E}_{K}\right)$ all lie in $L_{2}(G)$.

It is obvious from its definition that $\Theta \neq 0$. Hence we can choose $\delta \in \mathcal{E}_{K}$ such that $\Theta_{\delta} \neq 0$. Let $V$ be the smallest closed subspace of $L_{2}(G)$ containing $\Theta_{D}$, which is invariant under the left-regular representation $\lambda$ of $G$. Then $V \neq\{0\}$ and it is easy to show that $V$ is the orthogonal sum of a finite number of subspaces which are all invariant and irreducible under $\lambda$. This shows that each of the corresponding irreducible representations belongs to the discrete series.

Define $\Theta_{\hat{a}}=0$ if $\hat{a}$ is a singular element of $\hat{A}$ and let $\mathfrak{S}$ be the smallest closed subspace of $L_{2}(G)$ which contains every $C^{\infty}$ eigenfunction of 8 lying in $L_{2}(G)$. For any $f \in C_{c}^{\infty}(G)$ and $x \in G$, let $f_{x}$ denote the function $y \rightarrow f(y x)(y \in G)$.

TheOREM 4. The series

$$
\sum_{\hat{a} \in \hat{A}} \Theta^{\wedge}(f) \quad\left(f \in C_{c}^{\infty}(G)\right)
$$

converges absolutely and the function

$$
f^{\#}: x \rightarrow \sum_{\in \hat{A}} \theta_{\hat{a}}^{\hat{a}}\left(f_{x}\right)
$$

lies in $\mathfrak{S}$. Moreover the Haar measure of $G$ can be so normalized that $f-f^{\#}$ is orthogonal to $\mathfrak{S}$ for every $f \in C_{c}^{\infty}(G)$.

Theorem 4 shows that our method gives the entire discrete series.

5. The proofs of these results are rather long. We shall only give a brief outline of the main steps in the proofs of Theorems 1 and 2. As before, let $\mathfrak{g}_{c}$ be the complexification of the Lie algebra $\mathfrak{g}$ of $G$ and $S\left(\mathfrak{g}_{c}\right)$ the symmetric algebra over $\mathfrak{g}_{c}$. $G$ operates on $\mathfrak{g}_{c}$ by means of the adjoint representation. Let $I\left(g_{c}\right)$ be the subalgebra of all invariants of $G$ in $S\left(g_{c}\right)$. Now we take (in the set up of $\$ 1$ ) $M=g$ and $\omega$ the differential form corresponding to the Euclidean measure $d X$ on $\mathrm{g}$. For $p \in S\left(g_{c}\right)$, define the differential operator $\partial(p)$ on $g$ as in $[4, \S 2]$ and identify $\mathfrak{g}_{c}$ with its dual under the Killing form $\Omega$ given by $\Omega(X)$ $=\operatorname{tr}(\operatorname{ad} X)^{2}\left(X \in g_{c}\right)$. Let $\mathfrak{g}^{\prime}$ be the set of all regular elements of $\mathfrak{g}$. Then $\mathfrak{g}^{\prime}$ is open and dense in $\mathfrak{g}$ and its complement is of measure zero. 
A subset $U$ of $g$ is called completely invariant, if it satisfies the following condition. $C$ being any compact subset of $U, \operatorname{Cl}\left(C^{\theta}\right) \subset U$. Here $C^{G}=\cup_{x \in G} C^{x}$ and $\mathrm{Cl}$ denotes closure. If $U$ is an open and completely invariant subset of $\mathfrak{g}$, we can take $M=U$ in $\$ 1$.

LEMMA 1. Let $T$ be a distribution on a completely invariant open subset $U$ of $g$ such that:

(1) $T^{x}=T(x \in G)$,

(2) There exists an ideal $\mathfrak{U}$ in $I\left(\mathfrak{g}_{c}\right)$ such that $\operatorname{dim} I\left(\mathfrak{g}_{c}\right) / \mathfrak{U}<\infty$ and $\partial(u) T=0$ for $u \in \mathfrak{U}$.

Then $T$ is a locally summable function on $U$, which is analytic on $U^{\prime}=U \cap \mathfrak{g}^{\prime}$.

This is proved by induction on $\operatorname{dim} g$. Let $\Re$ be the set of all $X \in g$ such that $\operatorname{ad} X$ is nilpotent. The most important step in the proof of Lemma 1 is the following result.

LemMa 2. Let $T$ be an invariant distribution on $g$ such that ${ }^{4}$ Supp $T$ $\subset \Re$ and $\partial(\Omega) T=0$. Then $T=0$.

The proof of this makes use of a result of Kostant [6, Corollary 3.7 and Lemma 5.1] from which it follows (see $[2,2.3]$ ) that $\mathscr{X}$ is the union of a finite number of $G$-orbits.

In order to obtain Theorem 1, we have now to lift the result of Lemma 1 to the group. For this one needs the following fact.

Lemma 3. Let $D$ be a polynomial differential operator $[4, \S 2]$ on $\mathfrak{g}$ such that $D^{x}=D(x \in G)$ and $D p=0$ for $p \in I\left(g_{c}\right)$. Then $D T=0$ for every invariant distribution $T$ on $\mathfrak{g}$.

The proof again proceeds by induction on $\operatorname{dim} \mathrm{g}$. The crucial part is the following lemma.

Lemma 4. Let $T$ be a distribution and $D$ a polynomial differential operator on $\mathfrak{g}$. We assume that:

(1) $T^{x}=T(x \in G)$,

(2) $D^{x}=D$ and $D p=0\left(x \in G, p \in I\left(\mathfrak{g}_{0}\right)\right)$,

(3) Supp $D T \subset \Re$.

Then $D T=0$.

First one shows that it is sufficient to consider the case when $T$ is tempered. (This requires a result of Borel, according to which, we can always find a discrete subgroup $\Gamma$ of $G$ such that $G / \Gamma$ is compact. See Remark (2) at the bottom of p. 582 of [1].) Now we use the

\footnotetext{
${ }^{4}$ Supp $T$ denotes the support of $T$.
} 
theory of Fourier transforms. Put $B(X, Y)=\operatorname{tr}(\operatorname{ad} X$ ad $Y)(X, Y \in \mathfrak{g})$ and define

$$
\hat{f}(Y)=\int e^{i B(Y, X)} f(X) d X \quad\left(f \in C_{c}^{\infty}(\mathfrak{g}), Y \in \mathfrak{g}\right) .
$$

Then for any tempered distribution $\tau$, its Fourier transform is defined by $\hat{\tau}(f)=\tau(\hat{f})\left(f \in C_{c}^{\infty}(\mathfrak{g})\right)$. Let $J$ be the ideal of $I\left(\mathfrak{g}_{c}\right)$ spanned by all homogeneous elements of degree $\geqq 1$. Then $\Re$ is exactly the set of zeros of $J$ in $\mathfrak{g}$. Let $p_{1}, \cdots, p_{r}$ be an ideal basis for $J$. Then for every $j(1 \leqq j \leqq r)$, we can choose an integer $m_{j} \geqq 0$ such that $p_{j}^{m_{j}} D T=0$ around the origin. Since Supp $D T \subset \Re$ and $D T$ is invariant, it follows that $p_{j}^{m_{j}} D T=0$. Let $\mathfrak{U}$ be the ideal in $I\left(\mathfrak{g}_{c}\right)$ generated by $p_{j}^{m_{j}}(1 \leqq j \leqq r)$. Then $\operatorname{dim} I\left(\mathfrak{g}_{c}\right) / \mathfrak{U}<\infty$ and $u D T=0$ for $u \in \mathfrak{U}$. Hence we conclude from Lemma 1 that $(D T)^{-}$is a locally summable function. Now define $\hat{D}$ as in $[4, \mathrm{p} .91]$. Then $(D T)^{-}=\hat{D} \hat{T}$ and it is easy to see that $\hat{D}$ also verifies condition (2) of Lemma 4 . From this it follows without difficulty that $\hat{D} \sigma=0$ on $\mathfrak{g}^{\prime}$ for any invariant distribution $\sigma$ on $\mathfrak{g}$. Hence $\hat{D} \hat{T}=0$ on $\mathfrak{g}^{\prime}$. But since $\hat{D} \hat{T}$ is a locally summable function, this implies that $\hat{D} \hat{T}=0$ and therefore $D T=0$.

6. Now we come to Theorem 2. So assume that rank $\mathfrak{g}=\operatorname{rank}$ where $\mathfrak{t}$ is the Lie algebra of $K$. Put $\mathfrak{a}^{\prime}=\mathfrak{a} \cap \mathfrak{g}^{\prime}$ and $\pi=\prod_{\alpha \in P} \alpha$. Then $\pi$ is a polynomial function on $\mathfrak{a}_{6}$.

Lemma 5. Fix $H_{0} \in \mathfrak{a}^{\prime}$ and let $T$ be a tempered and invariant distribution on g such that

$$
\partial(p) T=p\left(i H_{0}\right) T \quad\left(p \in I\left(\mathfrak{g}_{c}\right)\right) .
$$

Then if $f^{5} T(H)=0$ for $H \in \mathfrak{a}^{\prime}$, we can conclude that $T=0$.

Lemma 6. Fix $H_{0} \in \mathfrak{a}^{\prime}$. Then there exists exactly one tempered and invariant distribution $T$ on $\mathfrak{g}$ such that:

(1) $\partial(p) T=p\left(i H_{0}\right) T \quad\left(p \in I\left(g_{c}\right)\right)$,

(2) $T(H)=\pi(H)^{-1} \sum_{s \in W} \epsilon(s) e^{i B\left(H_{0}, s H\right)} \quad\left(H \in \mathfrak{a}^{\prime}\right)$.

The uniqueness of $T$ follows from Lemma 5. The existence is proved as follows. Put

$$
\tau(f)=\pi\left(H_{0}\right) \sum_{\mathfrak{s} \in W} \int_{G} \hat{f}\left(\left(s H_{0}\right)^{x}\right) d x \quad\left(f \in C_{c}^{\infty}(\mathfrak{g})\right) .
$$

Then $\tau$ is a tempered and invariant distribution and $\partial(p) \tau=p\left(i H_{0}\right) \tau$

${ }^{5}$ In view of Lemma 1 , we can speak of the value $T(X)$ of $T$ at any point $X$ in $\mathfrak{g}^{\prime}$. 
for $p \in I\left(\mathfrak{g}_{c}\right)$ (see [5, pp. 225-226]). Moreover it can be shown that $\tau$ satisfies condition (2) of Lemma 6 up to a nonzero constant factor.

Theorem 2 is obtained by lifting the result of Lemma 6 to the group.

\section{REFERENCES}

1. A. Borel and Harish-Chandra, Arithmetic subgroups of algebraic groups, Bull. Amer. Math. Soc. 67 (1961), 579-583. $485-535$.

2. - Arithmetic subgroups of algebraic groups, Ann. of Math. (2) 75 (1962),

3. Harish-Chandra, On the characters of a semisimple Lie group, Bull. Amer. Math. Soc. 61 (1955), 389-396.

4. - Differential operators on a semisimple Lie algebra, Amer. J. Math. 79 (1957), 87-120.

5. - Fourier transforms on a semisimple Lie algebra. I, Amer. J. Math. 79 (1957), 193-257.

6. B. Kostant, The principal three-dimensional subgroup and the Betti numbers of a complex simple Lie group, Amer. J. Math. 81 (1959), 973-1032.

Columbia University

\section{CORRECTION TO ABSTRACT CLASS FORMATIONS ${ }^{1}$}

BY K. GRANT AND G. WHAPLES

Professor Yukiyosi Kawada has kindly pointed out to us that our construction for an abstract class formation $\{E(K)\}$ is wrong. Namely, we defined $E(K)$ to be a direct limit of a family of groups $\{M(K, N)\}$ under a mapping system $\left\{\eta_{N^{\prime} N}^{K}\right\}$. These maps $\eta_{N, N}^{K}$ induce on the second cohomology groups homomorphism whose kernel is not in general 0 ; hence it is in general not true that $H^{2}(F, E(k))$ $=Z(\# F) Z$. For details, see Theorem 2 of a paper by Kawada, forthcoming in Boletim da Sociedade de Matemática de São Paolo.

Our main theorem that a class formation does exist for every $G_{\infty}$, is however true: this is proved by Kawada in the paper just mentioned, using the same family of groups $M(K, N)$ but taking an inverse limit.

After seeing Kawada's work, one of us has found a correct construction using a direct limit and replacing the $\left\{\eta_{N, N}^{K}\right\}$ by a different system of maps. This will be explained in a paper to be published elsewhere.

Received by the editors September 11, 1962.

${ }^{1}$ Bull. Amer. Math. Soc. 67 (1961), 393-395. 